\title{
Deterministic Construction of Compressed Sensing Matrix Based on Q-Matrix
}

\author{
Yang Nie ${ }^{1,2}$, Xin-Le $\mathrm{Yu}^{1}$ and Zhan-Xin Yang ${ }^{1}$ \\ ${ }^{1}$ Digital Engineering Center, Communication University of China, Beijing, China \\ ${ }^{2}$ Department of Physics, Jining Normal University, Inner Mongolia, China \\ nieyangwork@163.com
}

\begin{abstract}
Compressed sensing is an innovative technology, which provides a new sampling mode. The key problem in compressed sensing is the construction of sensing matrix, which has an important influence on the signal sampling and reconstruction algorithm. At present, in most cases the sensing matrix is a random structure, and is difficult to realize due to its huge storage in practical applications. In this paper, we introduce a novel deterministic construction of sensing matrix via $\mathrm{Q}$-matrix, which is calculated by solving the N-queens problem. The proposed sensing matrix has good orthogonality and circularity. Using the circularity of Q-matrix, we can construct sensing matrix for compressed sensing. A large number of simulation results show that the proposed sensing matrix in this paper can obtain a better quality of the reconstructed image, and it is easily realized owing to its cyclic characteristic.
\end{abstract}

Keywords: Q-matrix; Compressed Sensing; Sensing Matrix; Image Compression

\section{Introduction}

Nyquist sampling theorem points out that if the sampling frequency is two times or more than the signal bandwidth, then the original signal or image can be reconstructed accurately from a small sample value. However, with data fast growing in the era of big data, signal bandwidth is also increased, which led to the sampling frequency continue to break through the bottleneck. Therefore the signal processing of large data is a difficult task. Compressed sensing (CS) [1-3] is a new form of signal sampling. Different from the traditional sampling theorem, the theory of CS emphasize that if the signal is compressible, then it can be used to convert to a low dimensional signal from the high dimensional signal, and the original signal can be reconstructed from a small number of low dimensional signals with high probability.

At present, the main research of CS includes mainly three aspects: the first aspect is to find a suitable sparse base for signal; the second concerns deterministic construction of sensing matrix; third is a fast and practical algorithm for signal reconstruction. Among them, the construction of sensing matrix is the most important in the study of CS. A stable sensing matrix can protect the salient information in any $\mathrm{k}$-sparse or compressible signal is not damaged by the dimensionality reduction.

The structure of the sensing matrix mainly includes two aspects: random sensing matrix and deterministic sensing matrix. The random structure of the sensing matrix has Gauss matrix and Bernoulli matrix. It can be proved that random matrices satisfy the Restricted Isometric Property (RIP) with high probability [4]. However, the random construction of sensing matrix is sometimes impractical to build in hardware. Conversely, the deterministic structure of the sensing matrix [5-9] has circulant matrix and Toeplitz matrix, which are easy to design because of less hardware. However, the typical deterministic matrices such as cyclic matrix and Toeplitz matrix still need to store lots of 
test data. Recently, the parity-check matrix of good LDPC codes can be used as provably good sensing matrix [10].

Inspired by the connection between LDPC codes and CS in, we construct the deterministic sensing matrix. The main contribution of this paper is that we propose a new deterministic construction of the sensing matrix by solving the $\mathrm{N}$-queens problem. Compared with other sensing matrix, the experimental simulation results show that the proposed sensor matrix not only has good reconstruction effect, but also is convenient for hardware implementation.

The rest of this paper is organized as follows. In Section 2, we review the basic theory of CS. The innovative construction method of sensing matrix based on Q-matrix is discussed in Section 3. In Section 4, several comparative experiments are conducted for the proposed method. Finally, discussion and conclusions are summarized in Section 5.

\section{Compressed Sensing}

All Compressed Sensing is a quite new framework that enables to get exact and approximate reconstruction of sparse or almost sparse signals from incomplete measurements. CS theory considers a $k$-sparse signal $x \in \square^{N}$ with $k$ nonzero elements, and then the system can get the measurements $y \in \square^{M}$ from linear projection in the noiseless setting.

$$
y=H x
$$

Where $H \in \square^{M \times N}$ is the sensing matrix with $M \square N$. Compressed sensing tries to recover the signal $\mathrm{x}$ from the measurement vector $\mathrm{y}$. The solution to this system by solving the following $l_{0}$-minimization problem

$$
\min \|x\|_{0} \quad \text { s.t. } \quad H x=y .
$$

However, it is well-known that (2) is NP-hard problem in general, which is a nonconvex optimization problem. There are two kinds of solution to recover the $k$-sparse signal $x$. The first method is greedy algorithms for $l_{0}$-minimization, such as orthogonal matching pursuit (OMP) [11], which can exactly recover $x$. The second method is to found convex relationship to (2), and $\mathrm{x}$ can recovered via $l_{1}$-minimization

$$
\min \|x\|_{1} \quad \text { s.t. } \quad H x=y .
$$

This solution to recover the $\mathrm{k}$-sparse signal $\mathrm{x}$ can be completed by the basis pursuit (BP) algorithm [12].

In compressive sensing, the structure of the sensing matrix is one of the main concerns. In order to measure the accuracy of the recovered signal and determine which sensing matrix is better, some criteria have been proposed. A meaningful and useful criteria called restricted isometry property (RIP) is put forward $[4,13]$. The standard specifies that if the matrix $H$ satisfies the RIP of order $k$ with enough small restricted isometry constant $\delta$, he $k$-sparse signal can be exactly recovered by $l_{0}$ or $l_{1}$ minimization. In addition, it is proposed a sufficient and necessary condition of exactly recovering, named the null space property (NSP) [14]. Although NSP and RIP all provide guarantees for the recovery of $k$-sparse signal, but they are very hard computable. In many cases it is preferable to use properties of $H$ that are easily computable to provide more concrete recovery conditions. 
The coherence of a matrix is one such property. The coherence of a matrix $H$, denoted $\mu(H)$, is the largest absolute inner product between any two columns $h_{i}, h_{j}$ of $H$ and is then defined as:

$$
\mu(H) \square \max _{1 \leq i \neq j \leq N} \frac{\left|\left\langle h_{i}, h_{j}\right\rangle\right|}{\left\|h_{i}\right\|_{2}\left\|h_{j}\right\|_{2}}
$$

Where $\left\langle h_{i}, h_{j}\right\rangle \sqcup h_{i}^{T} h_{j}$ denotes inner product of vectors. The following proposition bounds the value of the coherence for an arbitrary matrix. Let $H$ be a matrix of size $M \times N$ with $M<N$, whose columns are normalized so that $\left\|h_{i}\right\|=1$ for all $i$. Then the mutual coherence of A satisfies

$$
\sqrt{\frac{N-M}{M(N-1)}} \leq \mu(H) \leq 1
$$

The lower bound in (5) is known as the Welch bound [15]. If $N \square M$, the lower bound is approximately $\mu(H) \geq \sqrt{1 / M}$.

\section{Deterministic Construction of Sensing Matrix Based on Q-Matrix}

\subsection{The N-Queens Problem}

The 8-Queens puzzle [16] is the problem of putting eight chess queens on an $8 \times 8$ chessboard such that none of them is able to capture any other using the standard chess queen's moving. The queens must be placed in such a way that no two queens would be able to attack each other. Thus, a solution requires that no two queens share the same row, column, or diagonal. It has long been known that there are 92 solutions to the problem. All of the 92 solutions can be transformed into one of these 12 unique patterns using rotations and reflections and an arrangement of the 8-Queens is shown in Figure 1. If we increase the size of the chessboard beyond 8 rows/columns, we might want to find how many solutions exist for any arbitrary board size "N". The N-Queens puzzle is the problem of placing n queens on an $n$ chessboard such that no two queens attack each other. For example, if "N" $=10$, then there are 724 solutions. One of them is shown in Figure 2.

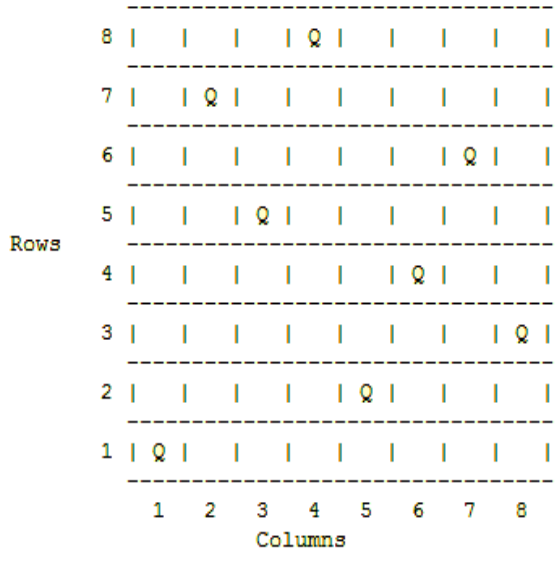

Figure 1. A Solution for 8-Queens

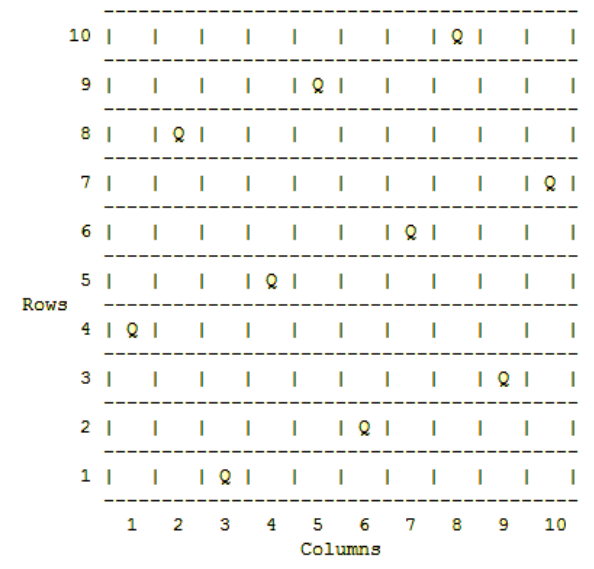

Figure 2. A Solution for 10-Queens 


\subsection{Q-matrix}

It can be seen from Figure 1 and Figure 2 that the solution of the $\mathrm{N}$-queens problem can be seen as a $n \times n$ matrix. If the number of 1's is only one in any a column, any a row or any a diagonal of it, a $n \times n$ non-diagonal identity square matrix is called the Q-matrix [17]. In generally, a Q-matrix is expressed by Q-vector. In this representation, the element position of the vector represents the column of the matrix, and the element value of the vector represents the row of non-zero elements in the matrix. Because the $n$ column (or row) vectors of the Q-matrix are linearly independent, so the Q-matrix is full rank matrix.

For example, we use the Q-matrix $(4 \times 4)$ to give a solution to the 4-Queens problem. Let $n=4$, using the four vectors of $(3,1,4,2)$, and $(1,4,2,3),(4,2,3,1)$ and $(2,3,1,4)$ to represent the corresponding Q-matrix. We obtain the following four matrices are shown in Figure 3. In Figure 3, it can be found that these four matrices are all satisfied with the definition of Q-matrix. That is there is only one " 1 " at each row, each column and each diagonal of them.

$$
Q_{1}^{4}=\left[\begin{array}{llll}
0 & 1 & 0 & 0 \\
0 & 0 & 0 & 1 \\
1 & 0 & 0 & 0 \\
0 & 0 & 1 & 0
\end{array}\right] Q_{2}^{4}=\left[\begin{array}{llll}
1 & 0 & 0 & 0 \\
0 & 0 & 1 & 0 \\
0 & 0 & 0 & 1 \\
0 & 1 & 0 & 0
\end{array}\right] Q_{3}^{4}=\left[\begin{array}{llll}
0 & 0 & 0 & 1 \\
0 & 1 & 0 & 0 \\
0 & 0 & 1 & 0 \\
1 & 0 & 0 & 0
\end{array}\right] \quad Q_{4}^{4}=\left[\begin{array}{llll}
0 & 0 & 1 & 0 \\
1 & 0 & 0 & 0 \\
0 & 1 & 0 & 0 \\
0 & 0 & 0 & 1
\end{array}\right]
$$

Figure 3. Q-matrix Based on Solving N-queens Problem

The Q-matrix has two important properties: circular shift and orthogonality. The cyclic shift property of the Q-matrix is convenient for hardware implementation. We assume that $W$ represents a set of Q-matrix, and $S$ represents a cyclic shift. $S_{t}^{j}(j=1,2, \cdots, n)$ indicates the number of cyclic shift and represents the type of cyclic shift (left, right). If a Q-matrix $Q \in W$, then $S^{j} Q \in W$. For example, $S_{\text {left }}^{1} Q_{1}^{4}=S_{\text {right }}^{3} Q_{2}^{4}$. Orthogonality is defined that the column or row vector of Q-matrix are linearly independent, and the transpose matrix of $\mathrm{Q}$ matrix is equal to its inverse matrix, i.e. $\left[Q_{1}^{4}\right]^{T}=\left[Q_{1}^{4}\right]^{-1}$. It can be also proved that any arbitrary $n$-dimensional Q-matrix is an orthogonal matrix.

\subsection{The Construction of Sensing Matrix Based on Q-matrix}

The sensing matrix is constructed by using the Q-matrix as the sub-matrix to block structure. The construction method of sensing matrix based on Q-matrix is as follows:

(1) We get an $n$-dimensional Q-matrix by solving the N-Queens problem. Backtracking search is frequently applied to solve the N-Queens problem. However, Suffering from exponential growth of computing time, backtracking search techniques are not able to solve it. In this paper, we use an effective local search with conflict minimization for solving the N-Queens problem [18]. The method does not need backtracking search, and its computation time is linear with $\mathrm{n}$.

(2) An $n$-dimensional matrix Q-matrix can be obtained by step (1). The rest of the Qmatrix is obtained by cyclic shift. If the $n$ is large enough, the number of Q-matrix can be guaranteed.

(3) Finally, the sensing matrix is obtained according to a certain permutation rule with Q-matrix .The sensing matrix is formed by the number of mutually orthogonal Q-matrix 
is arranged in accordance with certain rules. Meet the requirements of the arrangement method have the following several $(25 \times 25$ Q-matrix as an example):

Random Arrangement (RA): if the $25 \times 25$ Q-matrix is shifted by a cyclic, 25 kinds of new Q- matrix can be obtained. Then 25 kinds of new Q-matrix are randomly arranged to form a sensing matrix, which is just one of the ways, as shown in (6).

$$
H=\left[\begin{array}{ccccc}
Q_{4} & Q_{19} & Q_{18} & Q_{9} & Q_{24} \\
Q_{15} & Q_{2} & Q_{22} & Q_{6} & Q_{5} \\
Q_{11} & Q_{10} & Q_{20} & Q_{23} & Q_{12} \\
Q_{17} & Q_{16} & Q_{1} & Q_{13} & Q_{25} \\
Q_{14} & Q_{7} & Q_{8} & Q_{3} & Q_{21}
\end{array}\right]
$$

Sequence Arrangement (SA): if the $25 \times 25$ Q-matrix is shifted by a cyclic, 25 kinds of new Q- matrix can be obtained. Then the Q-matrix is arranged by subscript in order to form a sensing matrix, as shown in (7).

$$
H=\left[\begin{array}{lllll}
Q_{1} & Q_{2} & Q_{3} & Q_{4} & Q_{5} \\
Q_{6} & Q_{7} & Q_{8} & Q_{9} & Q_{10} \\
Q_{11} & Q_{12} & Q_{13} & Q_{14} & Q_{15} \\
Q_{16} & Q_{17} & Q_{18} & Q_{19} & Q_{20} \\
Q_{21} & Q_{22} & Q_{23} & Q_{24} & Q_{25}
\end{array}\right]
$$

Arithmetic Progression Arrangement (APA): if the $25 \times 25$ Q-matrix is shifted by a cyclic, 25 kinds of new Q-matrix can be obtained. The subscript is arranged in arithmetic progression, and tolerance is different for each row. The first row of tolerance is 2 , second for tolerance is 3, and so on. But the tolerances must be met as a prime number, as shown in (8). Because there are only 25 kinds of results of cyclic shift for Q-matrix, so $Q_{28}=Q_{3}, Q_{35}=Q_{10}, Q_{33}=Q_{8}, Q_{44}=Q_{9}$ and $Q_{55}=Q_{5}$.

$$
H=\left[\begin{array}{lllll}
Q_{2} & Q_{4} & Q_{6} & Q_{8} & Q_{10} \\
Q_{3} & Q_{6} & Q_{9} & Q_{12} & Q_{15} \\
Q_{5} & Q_{10} & Q_{15} & Q_{20} & Q_{25} \\
Q_{7} & Q_{14} & Q_{21} & Q_{28} & Q_{35} \\
Q_{11} & Q_{22} & Q_{33} & Q_{44} & Q_{55}
\end{array}\right]
$$

Dimakis [10] showed that the parity-check matrix of good LDPC codes can be used as provably sensing matrix s under basis pursuit if their girths are larger than four. That is, the number of " 1 " in common between any two columns can be no greater than 1 , which means there is no closed path of length 4 . We propose a combination arrangement (CA) to construct the sensing matrix by Q-matrix to avoid girth-4, and it can be expressed as

$$
H=\left[\begin{array}{lllll}
Q_{1} & Q_{1} & Q_{1} & Q_{1} & Q_{1} \\
Q_{1} & Q_{2} & Q_{4} & Q_{6} & Q_{8} \\
Q_{1} & Q_{3} & Q_{6} & Q_{9} & Q_{12} \\
Q_{1} & Q_{5} & Q_{10} & Q_{15} & Q_{20} \\
Q_{1} & Q_{7} & Q_{14} & Q_{21} & Q_{28}
\end{array}\right]
$$




\subsection{The Coherence of Sensing Matrix Based on Q-matrix}

To measure the performance of sensing matrices, RIP and NSP are the widely used criterions. But generally, there is no effective way to verify whether a sensing matrix satisfies RIP and NSP or not. As discussed in [17], any $k$-sparse signal can be exactly recovered from the measurement via BP algorithm or OMP, provided

$$
k<\frac{1}{2}\left[1+\frac{1}{\mu(H)}\right]
$$

Coherence is an important criterion to guarantee exact signal recovery, and one would design such that is minimized. The sensing matrix $H$ has $N$ columns $h_{1}, h_{2}, \ldots h_{N}$, and $w_{v}$ is the uniform column weight of the sensing matrix H. According to (4), $\left\|h_{i}\right\|_{2}=\sqrt{w_{v}}$ for $1 \leq i \leq N$. Since the proposed sensing matrix is free of cycles of length 4 ,so it is easy to see that any two distinct columns of $\mathrm{H}$ has only one same ' 1 ' in all lows, so the maximum inner product of any two columns is "1", So we have

$$
\mu(H)=\frac{1}{w_{v}}
$$

Another important criterion to measure the sensing matrix, the spark of the sensing matrix $\mathrm{H}$, is defined to be the smallest number of columns of $\mathrm{H}$ that are linearly dependent [17], can be calculated.

$$
\operatorname{spark}(H) \geq 1+\frac{1}{\mu(H)}=1+w_{v}
$$

To show the intuition for coherence of the proposed sensing matrix, Five types of matrices are generated as contrast: Gauss matrix, Toeplitz matrix and sparse random matrix with size $m \times n$, where $m=128$, and $n=256$. Assumed the column weight $w_{v}=4$ for the proposed sensing matrix and sparse random matrix. The mutual coherence of these matrices is plotted in Figure 4. It can be seen that the mutual coherence of the proposed sensing matrix is superior to other matrices.

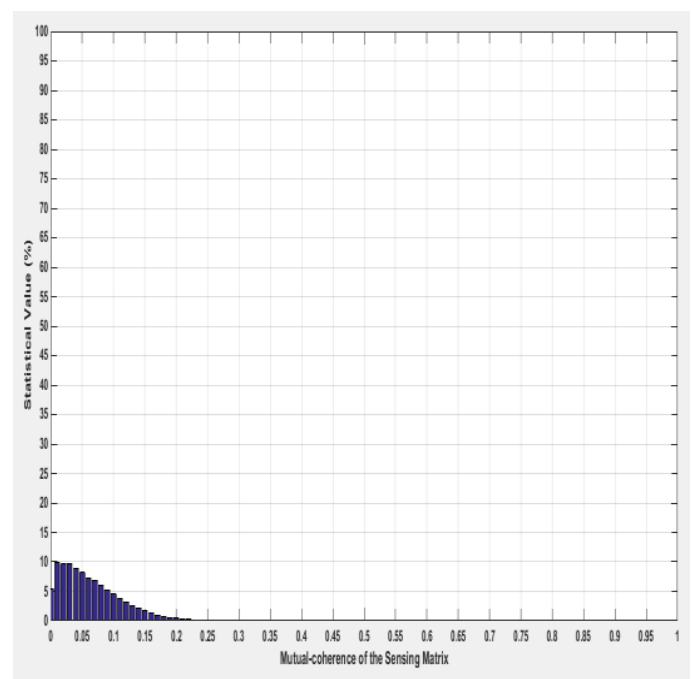

(a) The Coherence of Gauss matrix

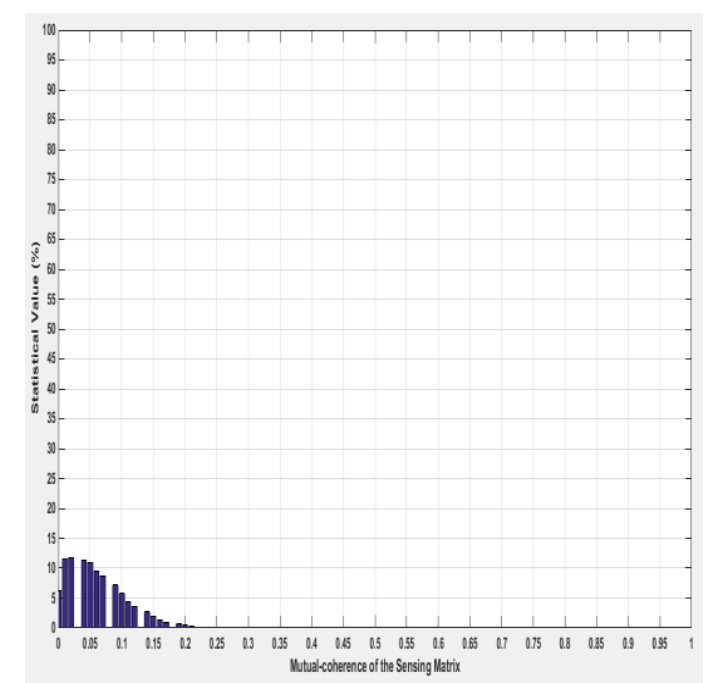

(b) The Coherence of Toeplitz Matrix 


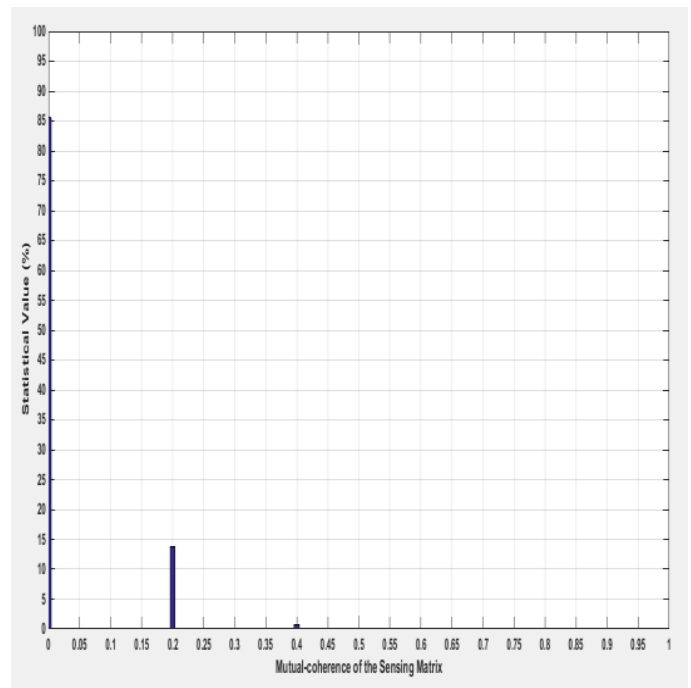

(c) The Coherence of Sparse Matrix

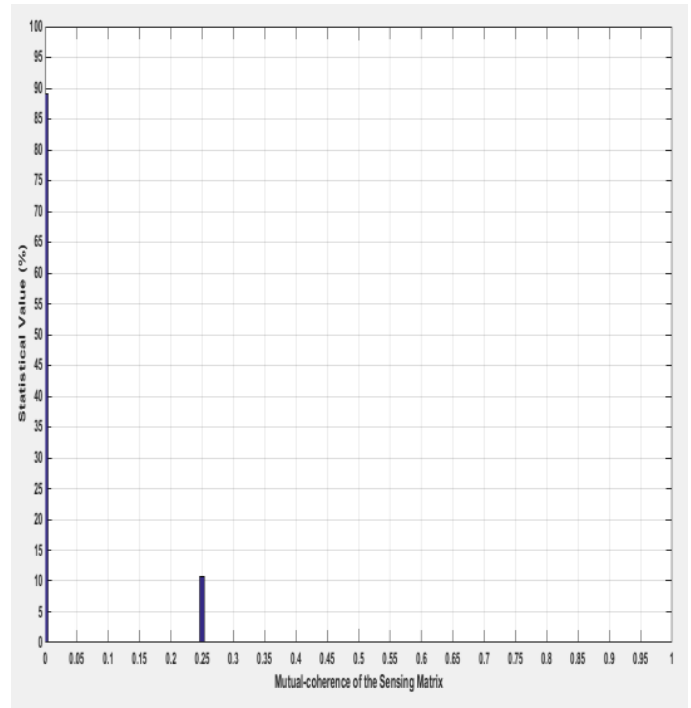

(e) The Coherence of Sensing Matrix (APA)

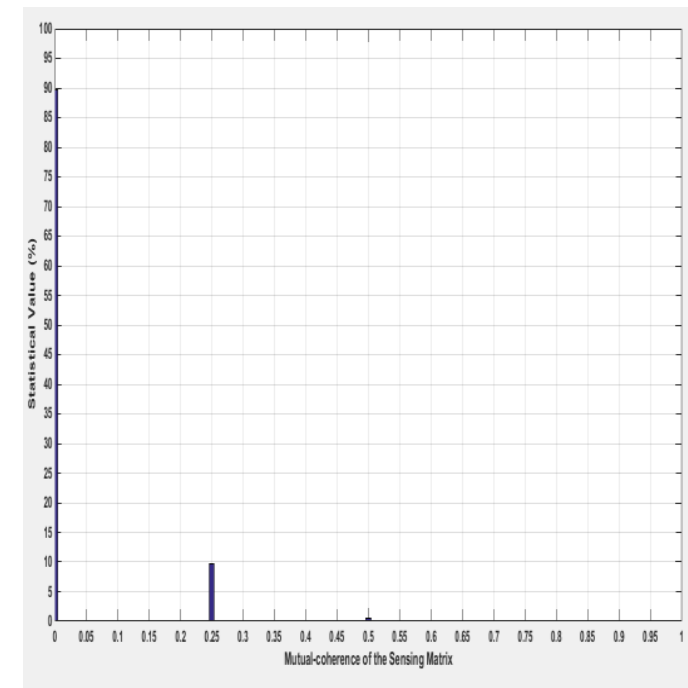

(d) The Coherence of Sensing Matrix (RA)

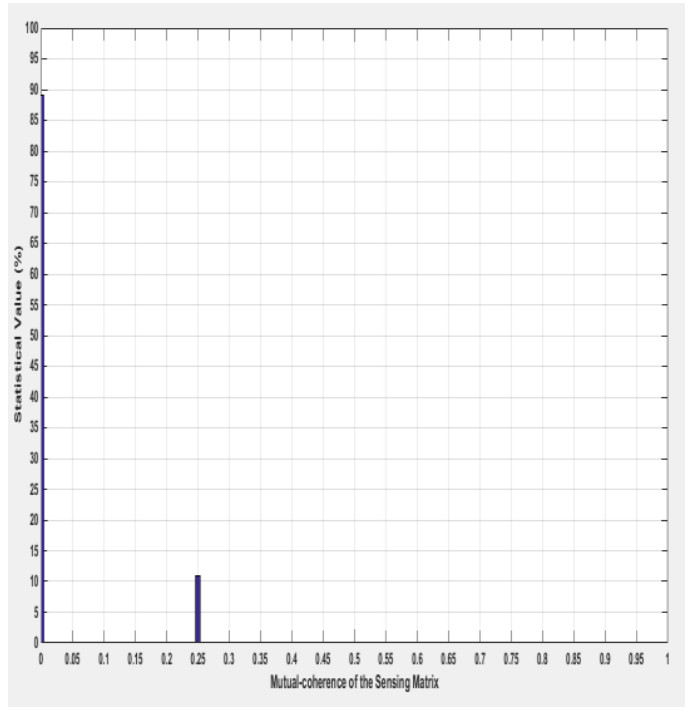

(f) The Coherence of Sensing Matrix (CA)

Figure 4. The Coherence of various Sensing Matrices

\section{Comparison of Experimental Results}

In this section, some simulation experiments are carried out to verify the performance of the proposed sensing matrix. In order to compare the results, the Gauss matrix and sparse random matrices are also designed for the sensing matrix. We give a $k$-sparse signal $x$ with length- $\mathrm{N}$, and $k$ is randomly generated. Then, the Gauss matrix, sparse random matrix, and the constructed sensing matrix are chosen as the sensing matrix to measure the signal $x$. Among them, the Gauss matrix is drawn with each entry is constructed and the columns of the matrix are normalized to unit magnitude. Sparse random matrix is constructed with a random way and its column weight is 5 . We use the Q-matrix to build the sensing matrix. In the process of the experiment, the size of the proposed detection matrix is $180 \times 360(M=180, N=360)$, and its column weight is 5 . The girth length of the proposed sensing matrix is 6 . According to (1), the measurements $y$ can be calculated. The recovery signal of $x$ from $y$ is done by the OMP algorithm.

The experiment was repeated 1000 times in each sparse level, and the probability of accurate recovery was calculated. In order to calculate the exact recovery of the 
frequency, the threshold $\left\|x^{\prime}-x\right\|_{2}<10^{-6}$ was used in the experimental process. The nonzero value of $k$-sparse signals is get according to a standard Gaussian distribution. It can be seen from Figure 5, the sensing matrix based on the Q-matrix can improve significantly the exact recovery probabilities in both data models compared to Gauss matrix, sparse random matrix.

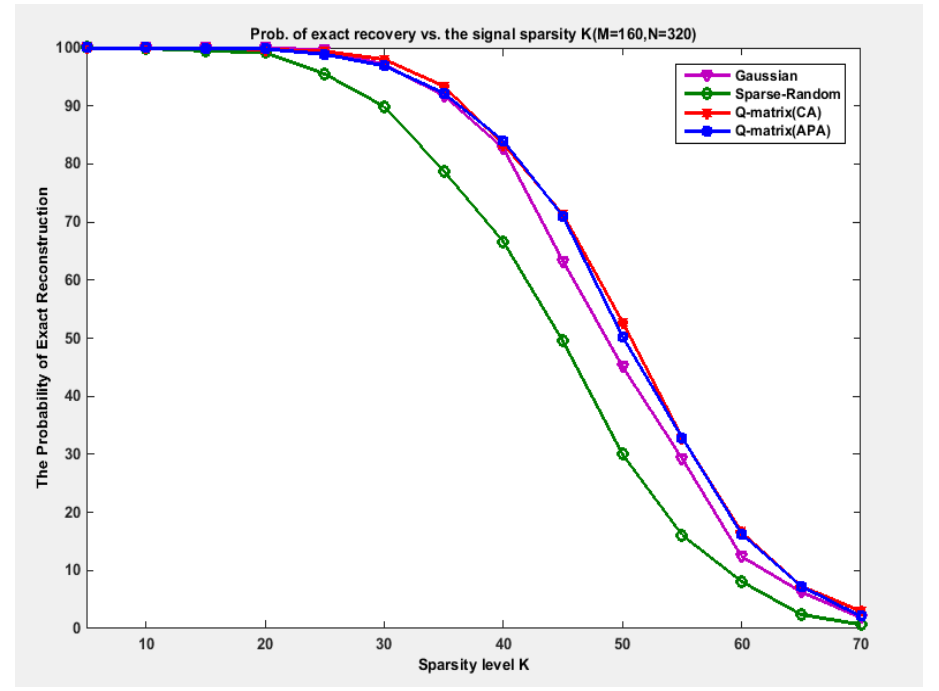

Figure 5. The Reconstitution Performance of Various Sensing Matrices (Column weight is 5)

To test the two-dimensional image signal of the proposed sensing matrix, the Lena image $(128 \times 256, M=128, N=256)$ is selected as the testing object. Compression ratio $(M / N)$ is 0.5 , and the reconstruction algorithm still uses the OMP algorithm. Gauss matrix, sparse random matrix, Toeplitz matrix, Bernoulli matrix and the proposed matrix are chosen as the sensing matrix. The column weight of the proposed sensor matrix is 4 , and the girth length is $10 \mathrm{In}$ order to compare the reconstruction performance of the matrix, Peak Signal to Noise Ratio (PSNR) and Mean Square Error (MSE) are used to measure the effect of reconstruction. When $x$ is the original signal and $x$ is the reconstructed signal, the calculation method of PSNR and MSE are as follows:

$$
\begin{gathered}
M S E=\frac{\sum_{i=0}^{N-1} \sum_{j=0}^{M-1}\left(x-x^{\prime}\right)^{2}}{M \times N} \\
P S N R=10 \lg \left(\left|\frac{255 \times 255}{M S E}\right|\right)
\end{gathered}
$$

As can be seen from Table 1, the proposed sensor matrix based on Q-matrix (arithmetic progression arrangement (APA) and combination arrangement (CA) compared with the other matrix, the value of PSNR has increased, and MSE also has a certain degree of improvement. In Fig. 6, the effect of different matrices in the same compression ratio is presented for the reconstruction of 2D image. Among them, the reconstruction effect of the proposed sensing matrix is better than that of the other sensing matrices. 
Table 1. The Performance Comparison of Different Sensing Matrices

\begin{tabular}{|c|c|c|}
\hline The Sensing Matrix & $\begin{array}{c}\text { Peak Signal to Noise Ratio } \\
\text { (PSNR) }\end{array}$ & $\begin{array}{c}\text { Mean Square Error } \\
\text { (MSE) }\end{array}$ \\
\hline Gauss matrix & 26.3281 & 151.4513 \\
\hline sparse random matrix & 26.2970 & 152.5401 \\
\hline Toeplitz matrix & 26.8336 & 134.8101 \\
\hline the proposed matrix(CA) & 26.8673 & 133.7680 \\
\hline the proposed matrix(APA) & 26.6516 & 140.5804 \\
\hline
\end{tabular}

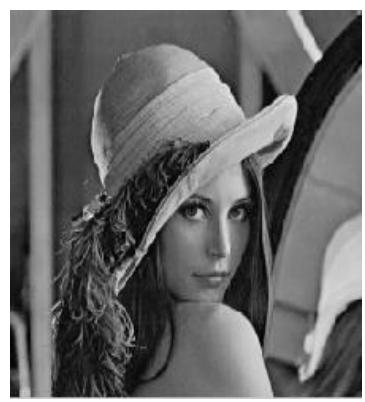

(a) Original Image

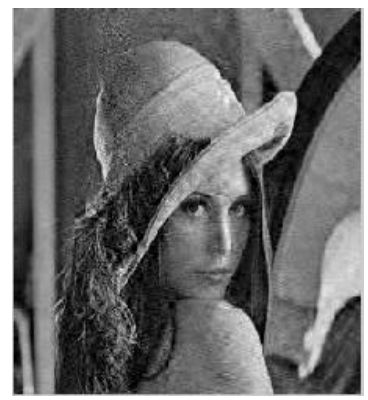

(d) Gauss Matrix

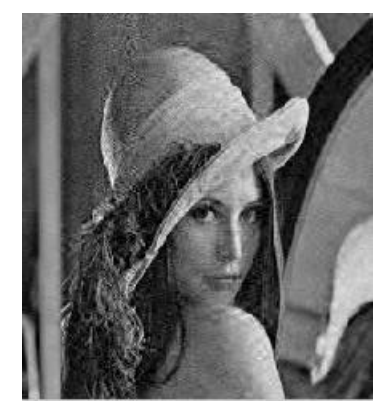

(b) The Proposed Matrix (CA)

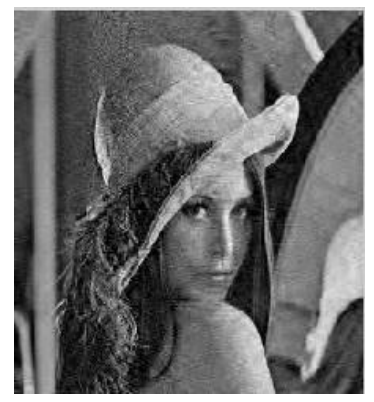

(e) Sparse Random Matrix

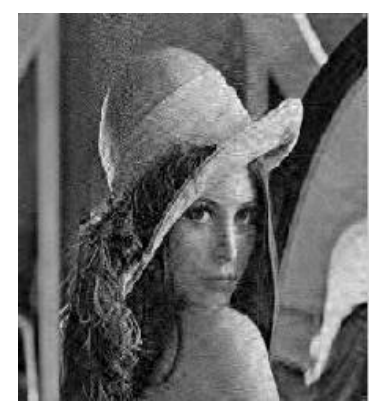

(c) The Proposed Matrix (APA)

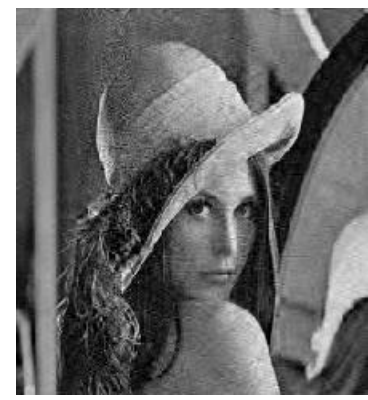

(f) Toeplitz Matrix

Figure 6. The Reconstruction Lena Images Using Five Sensing Matrix

\section{Conclusion}

In this paper we introduce a novel approach to construct deterministic sensing matrices based on Q-matrix. The coherence is used to analyze the optimal performance of the constructed sensing matrix. In addition, we set up the relationship between the column weight of a matrix and the coherence, and obtained the increase of the column weight to reduce the coherence. As compared with the existing constructions, the proposed sensing matrix is not only obtains better performance but also has easy hardware implementation.

\section{Acknowledgments}

We would like to thank professor Yang for stimulating discussions with respect to the topic of this paper and laboratory equipment. Moreover, we greatly appreciate the reviewers' comments that lead to an improved presentation of the results.

\section{References}

[1] E. J. Candes, J. Romberg, T. Tao, "Robust uncertainty principles: exact signal reconstruction from highly incomplete frequency information", IEEE Transactions on Information Theory, vol. 52, no. 2, (2006), pp. 489-509. 
[2] D. L. Donoho, "Compressed sensing", IEEE Transactions on Information Theory, vol. 52, no. 4, (2006), pp. 1289-1306.

[3] E. J. Candes, T. Tao, "Near-Optimal Signal Recovery From Random Projections: Universal Encoding Strategies?", IEEE Transactions on Information Theory, vol. 52, no. 12, (2006), pp. 5406-5425.

[4] E. J. Candes, T. Tao, "Decoding by linear programming", IEEE Transactions on Information Theory, vol. 51, no. 12, (2005), pp. 4203 - 4215.

[5] Lorne Applebaum, Stephen D. Howard, Stephen Searle, Robert Calderbank, "Chirp sensing codes: Deterministic compressed sensing measurements for fast recovery", Applied and Computational Harmonic Analysis, vol. 26, no. 2, (2009), pp. 283-290.

[6] Robert Calderbank, Stephen Howard, Sina Jafarpour, "Construction of a Large Class of Deterministic Sensing Matrices That Satisfy a Statistical Isometry Property", IEEE Journal of Selected Topics in Signal Processing, vol. 4, no. 2, (2010), pp. 358-374.

[7] Arash Amini, Farokh Marvasti, "Deterministic Construction of Binary, Bipolar, and Ternary Compressed Sensing Matrices", IEEE Transactions on Information Theory, vol. 57, no. 4, (2011), pp. 2360-2370.

[8] Arash Amini, Vahid Montazerhodjat, Farokh Marvasti, "Matrices With Small Coherence Using p-Ary Block Codes", IEEE Transactions on Signal Processing, vol. 60, no. 1, (2012), pp. 172-181.

[9] Shuxing Li, Fei Gao, Gennian Ge, Shengyuan Zhang, "Deterministic Construction of Compressed Sensing Matrices via Algebraic Curves", IEEE Transactions on Information Theory, vol. 58, no. 8, (2012), pp. 5035-5041.

[10] Alexandros G. Dimakis, Roxana Smarandache, Pascal O. Vontobel, "LDPC Codes for Compressed Sensing", IEEE Transactions on Information Theory, vol. 58, no. 5, (2012), pp. 3093-3114.

[11] Joel A. Tropp, Anna C. Gilbert, "Signal Recovery From Random Measurements Via Orthogonal Matching Pursuit", IEEE Transactions on Information Theory, vol. 53, no. 12, (2007), pp. 4655- 4666.

[12] Scott Shaobing Chen, David L. Donoho, Michael A. Saunders, "Atomic Decomposition by Basis Pursuit", Siam Review, vol. 43, no. 1, (2001), pp. 129-159.

[13] Richard Baraniuk, Mark Davenport, Ronald DeVore, Michael Wakin, "A Simple Proof of the Restricted Isometry Property for Random Matrices”, Constructive Approximation, vol. 28, no. 3, (2008), pp. 253263.

[14] M Stojnic, W Xu, B Hassib, "Compressed sensing-Probabilistic analysis of a null-space characterization", Proceedings of IEEE International Conference on Acoustics, Speech and Signal Processing, Las Vegas, Nevada, U.S.A., (2008), March 30-April 4, pp. 3377 - 3380.

[15] L. Welch, "Lower bounds on the maximum cross correlation of signals", IEEE Transactions on Information Theory, vol. 20, no. 3, (1974), pp. 397-399.

[16] R. Sosic, Jun Gu, “A polynomial time algorithm for the N-Queens problem”, ACM SIGART Bulletin, vol. 1, no. 3, (1996), pp. 7-11.

[17] L Peng, GX Zhu, "An Exploit of Designing Encoder for LDPC Codes Based on Q-matrix", Acta Electronica Sinica, vol. 33, no. 10, (2005), pp. 1734-1740.

[18] R. Sosic, Jun Gu, "Efficient local search with conflict minimization: a case study of the n-queens problem", IEEE Transactions on Knowledge and Data Engineering, vol. 6, no. 5, (1994), pp. 661-668.

\section{Author}

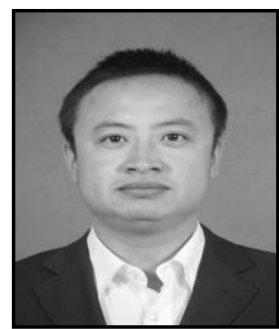

Yang Nie, he received the MS degree from Wuhan Research Institute of Posts \&Telecommunications in 2009. He is currently working toward the $\mathrm{PhD}$ degree in the Digital Engineering Center of Communication University of China. He is also a lecturer of Jining Normal University, China. His research interest includes Compressive Sensing, High-performance DSP Algorithms and VLSI Architectures. 